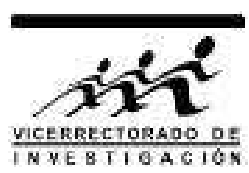

\title{
Resonancia ferromagnética en válvulas de espín: el caso del sistema $\operatorname{IrMn}(150 \AA) / \mathrm{Co}(50 \AA) / \mathrm{Ru}(32 \AA) / \mathrm{NiFe}(50 \AA)$
}

\author{
H. S. Tarazona Coronel, C. V. Landauro y J. Quispe Marcatoma \\ Facultad de Ciencias Físicas, Universidad Nacional Mayor de San Marcos, Ap. Postal 14-0149, Lima, Perú
}

Recibido 15 diciembre 2014 - Aceptado 10 enero 2015

\begin{abstract}
En el presente trabajo se hace un estudio teórico de la resonancia ferromagnética en una válvula de espín. Para ello, se propone un modelo fenomenológico de la densidad de energía libre de Helmholtz que toma en cuenta la anisotropía magnetocristalina, la anisotropía de forma, el acoplamiento de intercambio intercapa y el término conocido como exchange bias. Esta densidad de energía se usa para resolver la ecuación de Landau-Lifshitz en coordenadas esféricas y en condiciones de resonancia. Como resultado se obtiene la relación de dispersión, a partir de la cual se obtiene la frecuencia versus el campo resonante. Estos resultados están en buen acuerdo con lo publicado en la literatura. Adicionalmente, se aplica este modelo al sistema $\mathrm{IrMn} / \mathrm{Co} / \mathrm{Ru} / \mathrm{NiFe}$ para estudiar el acoplamiento intercapas magnéticas, donde la capa espaciadora, nomagnética, de rutenio tiene un espesor de $3.2 \mathrm{~nm}$. Los resultados indican la existencia de un acoplamiento antiferromagnético con una intensidad de acoplamiento $J_{b l}+2 J_{b c}=-4.3 \mathrm{erg} / \mathrm{cm}^{2}$.

Palabras claves: válvula de espín, resonancia ferromagnética, multicapa magnética, relación de dispersión.
\end{abstract}

\section{Ferromagnetic resonance of spin-valves: the case of $\operatorname{IrMn}(150 \AA) / \operatorname{Co}(50 \AA) / \operatorname{Ru}(32 \AA)$ $/ \mathrm{NiFe}(50 \AA)$ system}

In this work we study theoretically the ferromagnetic resonance in a spin-valve. For this purpose, we propose a phenomenological model for the density of Helmholtz free energy taking into account the magnetocrystalline anisotropy, shape anisotropy, interlayer exchange coupling and exchange bias. Considering this energy density we solve the Landau-Lifshitz equation in spherical coordinates and in the resonance condition. In this way, we obtain the dispersion relation and, as a consequence, the frequency versus the resonance field. These results are in good agreement with the literature. Additionally, we apply the model to the $\mathrm{IrMn} / \mathrm{Co} / \mathrm{Ru} / \mathrm{NiFe}$ system and study the magnetic coupling interlayers, where the non-magnetic layer (Ru) has a thickness of $3.2 \mathrm{~nm}$. The results indicate the existence of an antiferromagnetic coupling with a strength of couping of $J_{b l}+2 J_{b c}=-4.3 \mathrm{erg} / \mathrm{cm}^{2}$.

Keywords: Spin valve, ferromagnetic resonance, magnetic multilayer, dispersion relation.

La época en que vivimos está caracterizada por un avance acelerado que experimentan la ciencia y la tecnología. En el ámbito de la materia condensada la tendencia de investigación gira en torno de sistemas nanoscópicos ya que éstos sistemas presentan propiedades novedosas y de gran potencial tecnológico. Ese el caso de las multicapas magnéticas, especialmente de las válvulas de espín cuyas novedosas propiedades los hacen interesantes para la industria de los dispositivos de almacenamiento. En efecto, en 1991 Dieny y colaboradores [1] son los primeros en proponer el sistema válvula de espín para la fabricación de sensores magnéticos cuya primera aplicación fue como cabeceras lectoras de los discos duros [2]. Las válvulas de espín consisten de cuatro capas funcionales: dos capas ferromagnéticas (FM1 y FM2) separadas por una capa no magnética (NM) en la que la capa $\mathrm{FM} 2$ se encuentra en contacto con una capa antiferromagnética (AFM), la capa AFM sirve para fijar la magnetización de la capa FM2. Esta estructura da lugar a dos fenómenos muy importantes: el acoplamiento entre las capas ferromagnéticas llamado acoplamiento de intercambio intercapa y el acoplamiento Exchange bias producto del acople entre la capa ferromagnética y la capa antiferromagnética. Las propiedades magnéticas de este tipo de sistemas vienen siendo investigadas desde hace ya más de veinte años atras y aún no se logra entender completamente la física. Una de las técnicas

${ }^{*}$ heisembergtc@gmail.com 
con la que se estudia este tipo de sistema es la resonancia ferromagnética ya que ha demostrado ser una técnica que provee información cuantitativa y confiable de los materiales ferromagnéticos como son: el factor de Landé en los sólidos, las anisotropías magnéticas, el acoplamiento de intercambio intercapa y las cantidades relacionadas. En el presente trabajo se hace uso de la resonancia ferromagnética desde un enfoque teórico para estudiar el acoplamiento magnético en la válvula de espín.

Este artículo está organizado de la siguiente manera, en la próxima sección se indica el modelo fenomenológico de la densidad de energía libre y la relación de dispersión como solución de la ecuación de LandauLifshitz. En la siguiente sección se expone la relación entre la frecuencia versus el campo resonante y la dependencia angular del campo resonante, asimismo en una nueva sección se presenta la aplicación del modelo al sistema IrMn/Co/Ru/NiFe. Finalmente, las conclusiones se muestran al final del artículo.

\section{Modelo fenomenológico}

El sistema válvula de espín que se estudia en este trabajo se muestra ilustrativamente en la Fig. 1. Este sistema consiste de dos capas ferromagnéticas FM1 y FM2 separadas por un espaciador no magnético NM las cuales se encuentran sobre una capa antiferromagnética AFM. El sistema de coordenadas que se usa en este trabajo se muestra en la Fig. 2 en la que se asume que todas las capas permanecen en el plano $x-y$, siendo $M_{1}$ y $\boldsymbol{M}_{2}$ los vectores de magnetización de las capas FM1 y FM2, respectivamente, $\boldsymbol{M}_{3}$ la magnetización de una subred de la capa AFM, $\boldsymbol{H}$ el campo externo aplicado paralelo al plano de las capas y $\hat{\boldsymbol{\mu}}$ la dirección de anisotropía uniaxial para FM1 y FM2 a lo largo del eje $x$. A continuación se calculan las cantidades físicas de interés que permiten estudiar el comportamiento magnético de una válvula de espín.

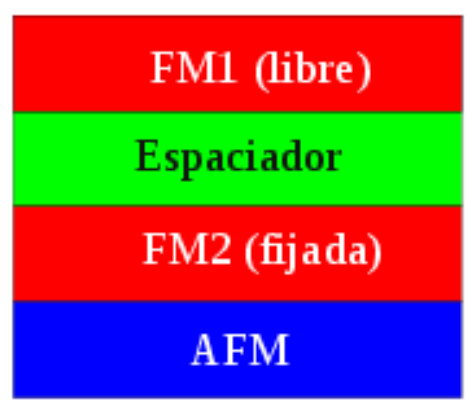

Figura 1: Diagrama esquemático de una válvula de espín.

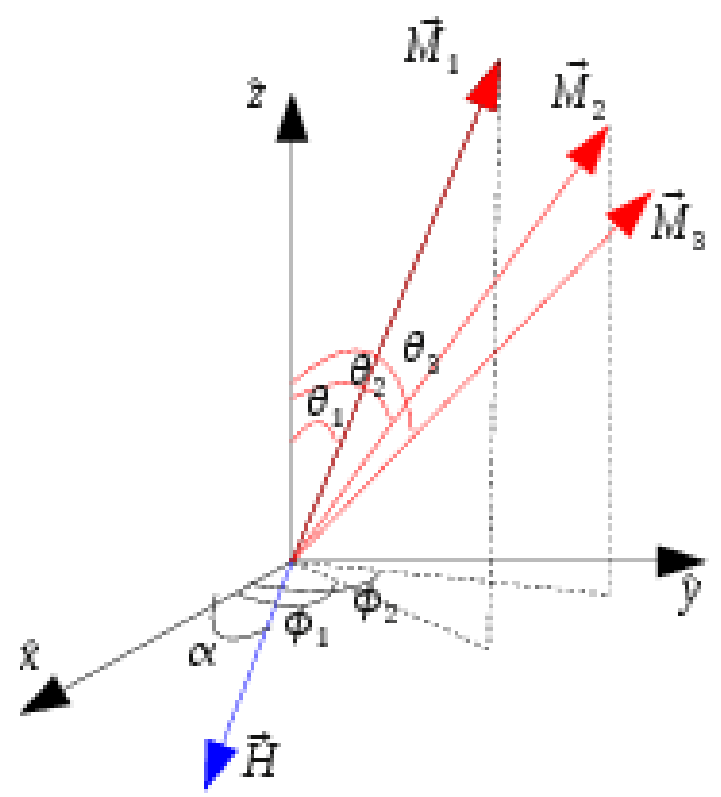

Figura 2: Sistema de coordenadas usado en el presente trabajo.

\section{Densidad de energía libre}

La densidad de energía libre de Helmholtz por unidad de superficie para la válvula de espín viene dada por

$$
E_{\text {total }}=E_{\text {Zee }}+E_{\text {form }}+E_{\text {anis }}+E_{\text {ex }}+E_{\text {acopl }},
$$

donde el primer término, $E_{\text {Zee }}$, representa la densidad de energía potencial de interacción entre la magnetización y el campo externo, energía de Zeeman; es decir,

$$
E_{\text {Zee }}=-t_{1} \boldsymbol{M}_{1} \cdot \boldsymbol{H}-t_{2} \boldsymbol{M}_{2} \cdot \boldsymbol{H},
$$

siendo $t_{1}$ y $t_{2}$ los espesores de las capas FM1 y FM2, respectivamente. El segundo término es la contribución de la energía proveniente de la anisotropía de forma; es decir,

$$
E_{\text {form }}=2 \pi t_{1}\left(M_{1} \cdot \hat{\boldsymbol{n}}\right)^{2}+2 \pi t_{2}\left(M_{2} \cdot \hat{\boldsymbol{n}}\right)^{2},
$$

siendo $\hat{\boldsymbol{n}}$ la normal al plano de las capas. El tercer término, $E_{\text {anis, }}$ es la contribución de la ansitropía magnetocristalina in-plane (en el plano) de las capas FM1, FM2 y viene dada por,

$$
E_{\text {anis }}=-K_{u 1} t_{1}\left(\frac{\boldsymbol{M}_{\mathbf{1}} \cdot \hat{\boldsymbol{\mu}}}{M_{1}}\right)^{2}-K_{u 2} t_{2}\left(\frac{\boldsymbol{M}_{2} \cdot \hat{\boldsymbol{\mu}}}{M_{2}}\right)^{2},
$$

donde $K_{u 1}, K_{u 2}$ son las constantes de anisotropía magnetocristalina uniaxial para las capas FM1 y FM2, respectivamente. El cuarto término, $E_{\mathrm{ex}}$, de la Ec. (1) viene a ser la contribución del intercambio de sesgo (exchange 
bias) que se da en la interface AFM-FM2 tal como fue planteado por Mauri et. al. [3], y viene dada por

$$
E_{\mathrm{ex}}=-\frac{J_{E} \boldsymbol{M}_{2} \cdot \boldsymbol{M}_{3}}{M_{2} M_{3}}-\sigma_{W} \frac{\boldsymbol{M}_{3} \cdot \hat{\boldsymbol{\mu}}}{M_{3}},
$$

donde $J_{E}$ es la constante de exchange bias efectiva y $\hat{\boldsymbol{\mu}}$ es la dirección de fácil magnetización de la capa AFM (los momentos que rotan rígidamente con FM2); tal que $H_{E}=J_{E} / M_{2} t_{2}$, representa al campo de exchange bias y el segundo término representa la energía de la pared de dominio planar en el que $\sigma_{W}$ es la energía de intercambio por unidad de superficie de una pared de dominio de $90^{\circ}$ en la capa AFM [4], siendo $H_{W}=\sigma_{W} / M_{2} t_{2}$ el campo de pared de dominio efectivo.

El quinto y último término en la Ec. 1] representa la ener- gía del acoplamiento de intercambio intercapa entre las capas ferromagnéticas FM1 y FM2, contribuyendo ya sea con una alineación bilineal o bicuadrática entre FM1 y FM2, cuya expresión fenomenológica viene expresada de la siguiente manera

$$
E_{\text {acopl }}=-J_{b l}\left(\frac{\boldsymbol{M}_{1} \cdot \boldsymbol{M}_{2}}{M_{1} M_{2}}\right)-J_{b c}\left(\frac{\boldsymbol{M}_{1} \cdot \boldsymbol{M}_{2}}{M_{1} M_{2}}\right)^{2},
$$

aquí $J_{b l}$ y $J_{b c}$ son las constantes de acoplamiento bilineal y bicuadrática entre las capas ferromagnéticas, respectivamente.

Ahora bien, teniendo en cuenta el sistema coordenado propuesto en el gráfico de la Fig. 2. la densidad de la energía libre de Helmholtz viene expresada tal como sigue,

$$
\begin{gathered}
E=t_{1}\left[-M_{1} H \operatorname{sen} \theta_{1} \cos \left(\alpha-\phi_{1}\right)+\left(2 \pi M_{1}^{2}-K_{u 1}^{\text {out }}\right) \cos ^{2} \theta_{1}-\frac{M_{1} H_{u 1}}{2} \operatorname{sen}^{2} \theta_{1} \cos ^{2} \phi_{1}\right] \\
+t_{2}\left[-M_{2} H \operatorname{sen} \theta_{2} \cos \left(\alpha-\phi_{2}\right)+\left(2 \pi M_{2}^{2}-K_{u 2}^{\text {out }}\right) \cos ^{2} \theta_{2}-\frac{M_{2} H_{u 2}}{2} \operatorname{sen}^{2} \theta_{2} \cos ^{2} \phi_{2}\right. \\
\left.-M_{2} H_{E}\left(\operatorname{sen} \theta_{2} \operatorname{sen} \theta_{3} \cos \left(\phi_{2}-\phi_{3}\right)+\cos \theta_{2} \cos \theta_{3}\right)-M_{2} H_{W} \operatorname{sen} \theta_{3} \cos \phi_{3}\right] \\
-J_{b l}\left[\operatorname{sen} \theta_{1} \operatorname{sen} \theta_{2} \cos \left(\phi_{1}-\phi_{2}\right)+\cos \theta_{1} \cos \theta_{2}\right] \\
-J_{b c}\left[\operatorname{sen} \theta_{1} \operatorname{sen} \theta_{2} \cos \left(\phi_{1}-\phi_{2}\right)+\cos \theta_{1} \cos \theta_{2}\right]^{2} .
\end{gathered}
$$

\section{La relación de dispersión}

Para determinar la relación de dispersión se resuelve la ecuación de Landau-Lifshitz [5], dada por

$$
\frac{\partial \vec{M}_{i}(t)}{\partial t}=-\gamma \vec{M}_{i}(t) \times \vec{H}_{e f f}
$$

en la que se ha omitido, por razones de simplicidad, el término de amortiguamiento [6]. Para resolver esta ecuación se utiliza el método de Smit y Beljers [7], el cual, en resumen, consiste en: (1) transformar la Ec. (8) a coordenadas esféricas, quedando dicha ecuación de la forma

$$
\frac{\partial \theta_{i}}{\partial t}=-\frac{\gamma}{t_{i} M_{i} \operatorname{sen} \theta_{i}} \frac{\partial E}{\partial \phi_{i}}
$$

$$
\frac{\partial \phi_{i}}{\partial t}=\frac{\gamma}{t_{i} M_{i} \operatorname{sen} \theta_{i}} \frac{\partial E}{\partial \theta_{i}},
$$

donde el campo efectivo ha sido expresado en términos de la densidad de energía $\boldsymbol{H}_{\mathrm{eff}}=\nabla_{M_{i}} E / t_{i} ;$ (2) expresar la densidad de energía $E$ en forma de una expansión en series de Taylor, tomando la aproximación a la segunda potencia; (3) en la condición de resonancia las desviaciones de las posiciones de equilibrio de la magnetización son muy pequeñas y son de la forma $\theta_{i}=\theta_{i}^{0}+\Delta \theta_{i} \exp (i \omega t)$ y $\phi_{i}=\phi_{i}^{0}+\Delta \phi_{i} \exp (i \omega t)$, donde $\theta_{i}^{0}$ y $\phi_{i}^{0}$ son las posiciones de equilibrio de las magnetizaciones de las capas FM1 y FM2 obtenidas al minimizar la Ec. (7).

Con estas tres consideraciones se resuelve la Ec. (9) y se obtienen seis ecuaciones acopladas las cuales pueden expresarse en forma matricial [8-10] tal como sigue

$$
\left[\begin{array}{cccccc}
\left(E_{\theta_{1} \phi_{1}}+i Z_{1}\right) & E_{\phi_{1} \phi 1} & E_{\phi_{1} \theta_{2}} & E_{\phi_{1} \phi_{2}} & E_{\phi_{1} \theta_{3}} & E_{\phi_{1} \phi_{3}} \\
E_{\theta_{1} \theta_{1}} & \left(E_{\theta_{1} \phi_{1}}-i Z_{1}\right) & E_{\theta_{1} \theta_{2}} & E_{\theta_{1} \phi_{2}} & E_{\theta_{1} \theta_{3}} & E_{\theta_{1} \phi_{3}} \\
E_{\theta_{1} \phi_{2}} & E_{\phi_{1} \phi_{2}} & \left(E_{\theta_{2} \phi_{2}}+i Z_{2}\right) & E_{\phi_{2} \phi_{2}} & E_{\phi_{2} \theta_{3}} & E_{\phi_{2} \phi_{3}} \\
E_{\theta_{1} \theta_{2}} & E_{\phi_{1} \theta_{2}} & E_{\theta_{2} \theta_{2}} & \left(E_{\theta_{2} \phi_{2}}-i Z_{2}\right) & E_{\theta_{2} \theta_{3}} & E_{\theta_{2} \phi_{3}} \\
E_{\theta_{1} \phi_{3}} & E_{\phi_{1} \phi_{3}} & E_{\theta_{2} \phi_{3}} & E_{\phi_{2} \phi_{3}} & \left(E_{\theta_{3} \phi_{3}}+i Z_{3}\right) & E_{\phi_{3} \phi_{3}} \\
E_{\theta_{1} \theta_{3}} & E_{\phi_{1} \theta_{3}} & E_{\theta_{2} \theta_{3}} & E_{\phi_{2} \theta_{3}} & E_{\theta_{3} \theta_{3}} & \left(E_{\theta_{3} \phi_{3}}-i Z_{3}\right)
\end{array}\right]\left[\begin{array}{c}
\Delta \theta_{1} \\
\Delta \phi_{1} \\
\Delta \theta_{2} \\
\Delta \phi_{2} \\
\Delta \theta_{3} \\
\Delta \phi_{3}
\end{array}\right]=0
$$


donde los elementos $E_{i j}$ denotan las segundas derivadas respecto a los ángulos $\theta_{i}$ y $\phi_{i}, Z_{i}=t_{i} \omega M_{i} \operatorname{sen} \theta_{i} / \gamma_{i}$ $(i=1,2,3)$, $\omega$ es la frecuencia de resonancia y $\gamma_{i}$ es la razón giromagnética. Para que esta ecuación matricial tenga solución no trivial, el determinante de la matriz debe ser cero, obteniendo así una ecuación característica

$$
\left(\frac{\omega}{\gamma}\right)^{4}-b\left(\frac{\omega}{\gamma}\right)^{2}+c=0,
$$

para $\gamma_{1}=\gamma_{2}=\gamma$. Esta ecuación es conocida como la relación de dispersión. Los coeficientes $b(H)$ y $c(H)$ se expresan como

$$
\begin{gathered}
b=\left(\frac{E_{\phi_{2} \phi_{3}}^{2} E_{\theta_{2} \theta_{3}}^{2}}{E_{\theta_{3} \theta_{3}} E_{\phi_{3} \phi_{3}}}-\frac{E_{\phi_{2} \phi_{2}} E_{\theta_{2} \theta_{3}}^{2}}{E_{\theta_{3} \theta_{3}}}-\frac{E_{\phi_{2} \phi_{3}}^{2} E_{\theta_{2} \theta_{2}}}{E_{\phi_{3} \phi_{3}}}\right) \times \\
\frac{1}{\left(t_{2} M_{2}\right)^{2}}+E_{\phi_{1} \phi_{1} \theta_{1} \theta_{1}} \times \frac{1}{\left(t_{1} M_{1}\right)^{2}}+2 E_{\theta_{1} \theta_{2}} E_{\phi_{1} \phi_{2}} \times \\
\quad\left(\frac{1}{t_{1} M_{1} t_{2} M_{2}}\right), \mathrm{y} \\
\left.\left(E_{\phi_{1} \phi_{2}}^{2}-E_{\phi_{1} \phi_{1}}\left(E_{\phi_{2} \phi_{2}}-\frac{E_{\phi_{2} \phi_{3}}^{2}}{E_{\phi_{3} \phi_{3}}}\right)\right)\right] \frac{1}{\left(t_{1} M_{1} t_{2} M_{2}\right)^{2}}
\end{gathered}
$$

respectivamente.

Para mayor detalles sobre la determinación de la relación de dispersión véase la Ref. [11].

\section{Resultados y discusión}

Para el estudio de la resonancia ferromagnética se ha resuelto numéricamente la Ec. (11) utilizando el algoritmo descrito en un trabajo previo, véase la Ref. [11]. Las consideraciones a hacer para resolver numéricamente esta ecuación son: (1) el campo externo $\boldsymbol{H}$ está dirigido a lo largo del eje uniaxial $\hat{u}$, donde $\alpha=0$, (2) existe un predominio del factor de acoplamiento bilineal $J_{b l}$ sobre el factor de acoplamiento bicuadrático $J_{b c}$ por lo que este último se desprecia, (3) la energía de rotación de la pared de dominio $\sigma_{W}$ se considera muy grande por lo que $H_{E} \ll H_{W}$. A partir de estas consideraciones se analizan los casos correspondientes al acoplamiento ferromagnético, $J_{b l}>0$, y antiferromagnético, $J_{b l}<0$, como se puede apreciar en las siguientes subsecciones.

\section{Acoplamiento ferromagnético $J_{b l}>0$}

En este tipo de acoplamiento, las magnetizaciones de las capas FM1 y FM2 son paralelas y tienen la misma dirección. La solución numérica indica que $\phi_{1}=\phi_{2}=\phi_{3}=$ $\alpha=0$ y se obtienen dos soluciones reales de la Ec. (11), las cuales son los dos modos normales de resonancia, como se puede apreciar en la Fig. 3 .

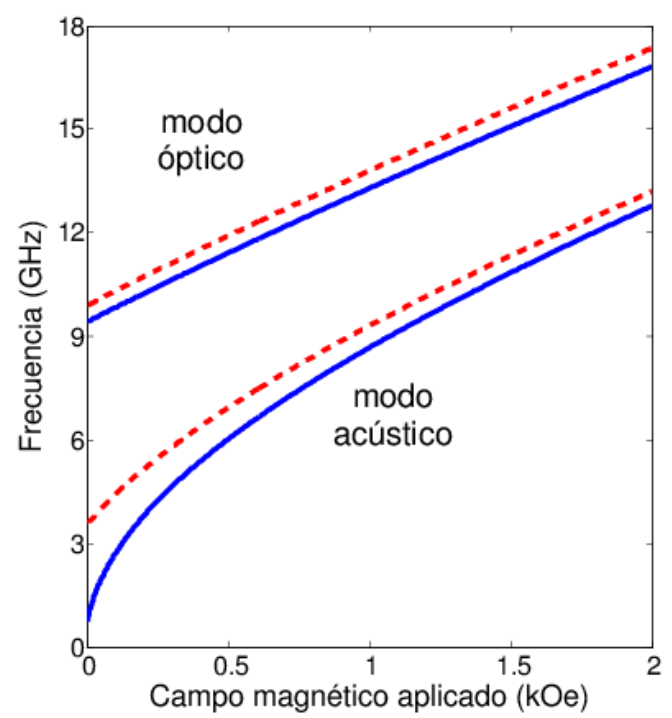

Figura 3: Relación entre la frecuencia de resonancia $f$ y el campo externo $H$, obtenidas a partir de la relación de dispersión (véase la Ec. 11), la cual fue simulada con los siguientes parámetros: $J_{b l}=0.5 \mathrm{erg} / \mathrm{cm}^{2}, J_{b c}=0 . H_{E}=0$ (líneas sólidas), $H_{E}=250$ Oe (líneas punteadas). Capa FM1: $4 \pi M_{1}=6$ kG, $H_{\text {kef } f_{1}}=-6 \mathrm{kOe}, H_{U 1}=0$ Oe, $t_{1}=100 \AA, \gamma_{1} / 2 \pi=2.8$ $\mathrm{GHz} \mathrm{kOe}-1$. Capa FM2: $4 \pi M_{2}=10 \mathrm{kG}, H_{k e f f_{2}}=-10 \mathrm{kOe}$, $H_{U 2}=10$ Oe, $t_{2}=200 \AA, \gamma_{2} / 2 \pi=2.8 \mathrm{GHzkOe}^{-1}$.

Así, el modo acústico consiste en una precesión en fase de las magnetizaciones $\boldsymbol{M}_{1}$ y $\boldsymbol{M}_{2}$, por lo que la energía de acoplamiento se mantiene constante durante la precesión sin afectar la condición de resonancia [12]. Por otro lado, el modo óptico consiste en la precesión desfasada de las magnetizaciones, alterando la energía de acoplamiento durante la precesión, desplazando así la curva hacia campos altos. Además, se puede observar que la aparición de un campo de anisotropía $H_{E}=250 \mathrm{Oe}$, líneas punteadas, ocasiona que la curva se desplace hacia altas frecuencias o campos bajos, siendo este corrimiento más importante para el modo acústico que para el modo óptico, tal como se discute con más detalle en la Ref. [13]. Adicionalmente, se ha hecho el estudio de cómo la fuerza de acoplamiento afecta a la relación de dispersión. Los resultados se muestran en la Fig. 4 donde se observa que el incremento de $J_{b l}$ ocasiona un corrimiento de los modos resonantes hacia frecuencias altas. 


\section{Acoplamiento antiferromagnético $J_{b l}<0$}

En este tipo de acoplamiento las magnetizaciones se alinean antiparalelamente en ausencia de un campo externo $(H=0)$, pero conforme el campo externo aumenta, las magnetizaciones tienden a alinearse paralelamente. La Fig. 5] muestra la relación de dispersión para $J_{b l}=-2$ erg $\mathrm{cm}^{-2}$ y es interesante observar que a diferencia del acoplamiento ferromagnético, el modo acústico corresponde a altas frecuencias o campos bajos y el modo óptico co-

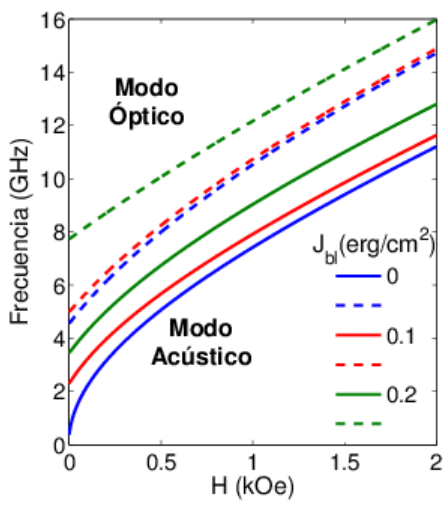

Figura 4: Dependencia entre la frecuencia $f$ y el campo $H$ resonante para distintos valores del factor de acoplamiento $J_{b l}$. Las líneas sólidas (modo acústico) y punteadas (modo óptico) del mismo color corresponden a un solo valor de $J_{b l}$ indicado en la leyenda. Los parámetros utilizados para la simulación fueron los mismos que el de la figura 3 con $H_{E}=0$.

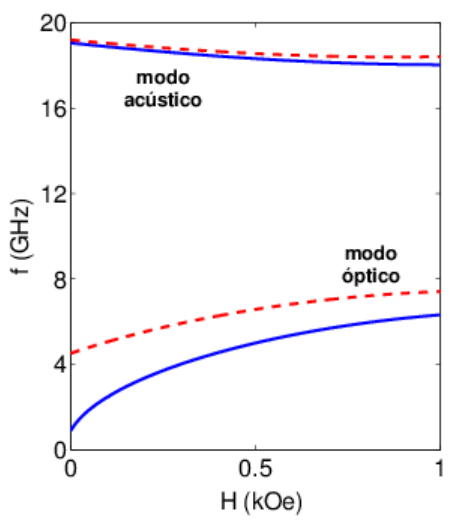

Figura 5: Relación de dispersión de un acoplamiento antiferromagnético para $J_{b l}=-2.0 \mathrm{erg} / \mathrm{cm}^{2}$. Los parámetros para la simulación fueron los mismos que se usaron en la figura 3 con $H_{E}=0$ (líneas sólidas) y $H_{E}=250$ Oe (líneas punteadas) rresponde a frecuencias bajas o campos altos [14,15. Asimismo, la aparición del término exchange bias $\left(H_{E}=250\right.$ Oe, líneas punteadas) parece afectar más al modo acústico que al modo óptico, observándose un gap de frecuencias. Por otro lado, en la Fig. 6] se observa la relación de dispersión para valores diferentes de $J_{b l}<0$. En ella se ve que para acoples débiles hay un predominio del modo acústico. Al contrario, para un acople AFM fuerte $\left(J_{b l}=5.0\right.$ $\mathrm{erg} / \mathrm{cm}^{2}$ ) hay mayor presencia del modo óptico.

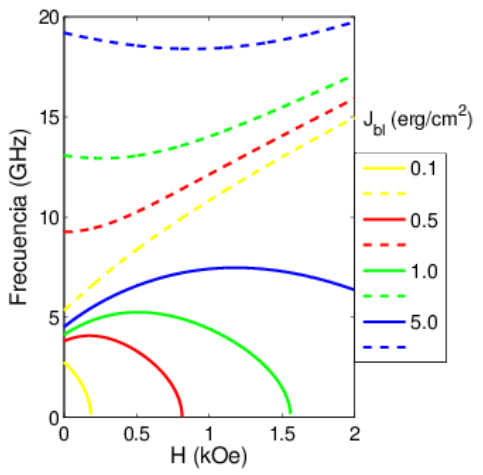

Figura 6: Relación de dispersión para diferentes valores del factor de acoplamiento $J_{b l}$ antiferromagnético. Las líneas sólidas (modo óptico) y punteadas (modo acústico) de un mismo color corresponden al mismo valor de $J_{b l}$. Los parámetros usados en la simulación fueron las mismas que el de la figura 3 con $H_{E}=250 \mathrm{Oe}$

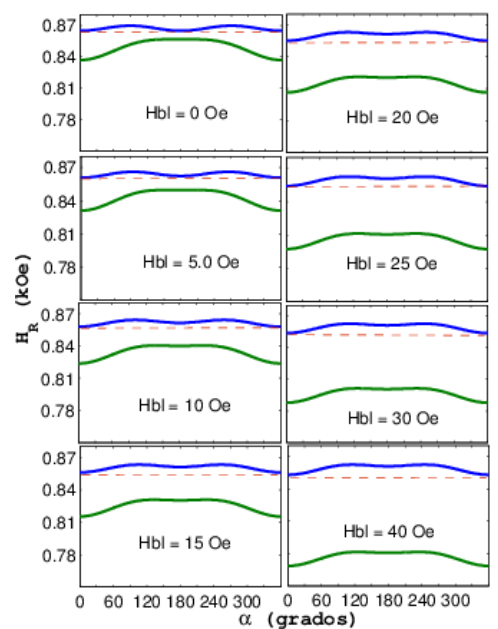

Figura 7: Dependencia angular $(\alpha)$ del campo resonante $\left(H_{R}\right)$ para diferentes valores del campo de acoplamiento bilineal $\left(H_{b l}\right)$. La línea azul corresponde al modo acústico y la línea verde al modo óptico. Los parámetros para la simulación fueron: $H_{u 1}=H_{u 2}=3.0 \mathrm{Oe}, 4 \pi M_{1}=10 \mathrm{kOe}, 4 \pi M_{2}=10.3$ $\mathrm{kOe}, t_{1}=t_{2}=100 \AA, H_{E}=0.010 \mathrm{kOe}, H_{u 1}^{\text {out }}=H_{u 2}^{\text {out }}=0.0$ $\mathrm{kOe}, J_{b c}=0$. 


\section{Efecto del factor de acoplamiento sobre la de- pendencia angular del campo resonante $H_{R}$}

Por lo general, en los experimentos de resonancia ferromagnética se mantiene constante la frecuencia de la radiación de microonda $\omega$ y se varía el campo externo $H$, en esta sección se muestran algunos resultados referentes a la dependencia angular del campo resonante. Para ello, se ha utilizado un algoritmo de tipo regresivo desarrollado en un trabajo previo [11], el cual consiste básicamente en la búsqueda de un valor de $H$ que satisfaga la relación de dispersión (Ec. (11)) manteniendo fija la frecuencia de radiofrecuencia $\omega$.

Este proceso se realiza para todos los ángulos en el rango, $0 \leq \alpha \leq 2 \pi$, con $\Delta \alpha=1^{\circ}$. El factor de acoplamiento bilineal $J_{b l}$ se ha simulado considerando un campo de acoplamiento bilineal $H_{b l}=J_{b l} / t_{i} M_{i}$, para cada capa FM $(i=1,2)$. Los resultados se muestran en la Fig. 7 Allí se puede observar que para $H_{b l}=0$ (sin acoplamiento) la curva de resonancia presenta una simetría de dos pliegues (twofold) lo cual es característico de estructuras cristalinas del tipo hexagonal de cuerpo centrado (hcp), y a medida que $H_{b l}$ se incrementa dicha simetía desaparece dando lugar a un rompimiento de simetría lo cual indica la aparición de un campo adicional producto del acoplamiento entre las capas FM1 y FM2.

Otra característica que se puede observar en la Fig. 7 es que a medida que el campo $H_{b l}$ se incrementa, los campos resonantes correspondientes al modo óptico y al modo acústico se desplazan hacia campos menores siendo este desplazamiento más pronunciado para la capa libre FM1. Finalmente, cabe mencionar que estos resultados están en buen acuerdo con los reportados en la Ref. [16].

\section{Aplicación del modelo al sistema $\operatorname{IrMn} / \mathrm{Co} / \mathrm{Ru}(\mathrm{t}=32 \AA) / \mathrm{NiFe}$}

Para demostrar la aplicación de este modelo a sistemas reales, empleamos los resultados experimentales obtenidos de muestras del sistema $\operatorname{lmn} / \mathrm{Co} / \mathrm{Ru}(\mathrm{t}=32 \AA) / \mathrm{NiFe}$, este estudio experimental escapa de los objetivos del presente trabajo. Sin embargo, brevemente se puede indicar que el sistema multicapa fue preparado empleando un magnetrón sputtering del tipo planar a temperatura ambiente y sobre un substrato de $\mathrm{Si}(111)$ cristalino.

Correspondientemente, las mediciones de FMR fueron hechas empleando un espectrómetro Bruker ESP3000 de alta sensibilidad con una frecuencia de microonda en la banda $X(9.79 \mathrm{GHz})$. Los detalles experimentales se indican en la Ref. [17]. En particular, el sistema estudiado en el presente trabajo es la multicapa $\mathrm{Si} / \operatorname{Ru}(150 \AA) / \operatorname{IrMn}(150 \AA) / \mathrm{Co}(50 \AA) / \operatorname{Ru}(\mathrm{t}=32 \AA) /$ $\mathrm{NiFe}(50 \AA) / \operatorname{Ru}(50 \AA)$.

La información concerniente al sistema $\left(\operatorname{lr}_{20} \mathrm{Mn}_{80}\right) / \mathrm{Co} / \mathrm{Ru}(32) / \mathrm{Ni}_{81} \mathrm{Fe}_{19}$ con la que se hizo la simulación se resume en la Tabla 1, A continuación se describe el proceso de ajuste del modelo a los datos experimentales del sistema bajo estudio.

\begin{tabular}{ccccc}
\hline Capa & $\begin{array}{c}\mathrm{t} \\
(\AA)\end{array}$ & $\begin{array}{c}H_{u} \\
(\mathrm{Oe})\end{array}$ & $\begin{array}{c}4 \pi M_{s} \\
(\mathrm{kOe})\end{array}$ & $\begin{array}{c}\gamma / 2 \pi \\
(\mathrm{GHz} / \mathrm{kOe})\end{array}$ \\
\hline $\mathrm{NiFe}$ & 50 & 3 & 9.8 & 2.8 \\
$\mathrm{Ru}$ & 32 & - & - & - \\
$\mathrm{Co}$ & 50 & 80 & 17.0 & 2.8 \\
IrMn & 150 & - & - & -
\end{tabular}

Tabla 1: En esta tabla se indican los valores del espesor $(t)$, campo uniaxial $\left(H_{u}\right)$ y magnetización de saturación $\left(M_{s}\right)$ correspondientes a cada capa de la válvula de espín. (Los datos fueron tomados de la Ref. [17] donde se estudia el mismo sistema en forma experimental).

Para empezar, cabe mencionar que en el experimento de resonancia ferromagnética el campo externo $H$ se ha aplicado paralelo al plano de las capas, por lo que las magnetizaciones también permanecerán en el plano de las capas $\left(\theta_{1}=\theta_{2}=\theta_{3}=90^{\circ}\right)$ reduciendo la densidad de energía $E$ (véase la Ec. (7)) a una expresión más sencilla, quedando las posiciones de la magnetización en función de los ángulos $\phi_{i}$. Bajo esa condición se establecen valores iniciales para los siguientes parámetros: campo de exchange bias $H_{E}$, razones giromagnéticas de ambas capas FMs $\gamma_{1}$ y $\gamma_{2}$, y campos uniaxiales para ambas capas $H_{u 1}, H_{u 2}$. Se ha mantenido constante las magnetizaciones de saturación tanto para la capa de $\mathrm{NiFe}\left(4 \pi M_{s}=10\right.$ kOe) como para el Co $\left(4 \pi M_{s}=17 \mathrm{kOe}\right)$.

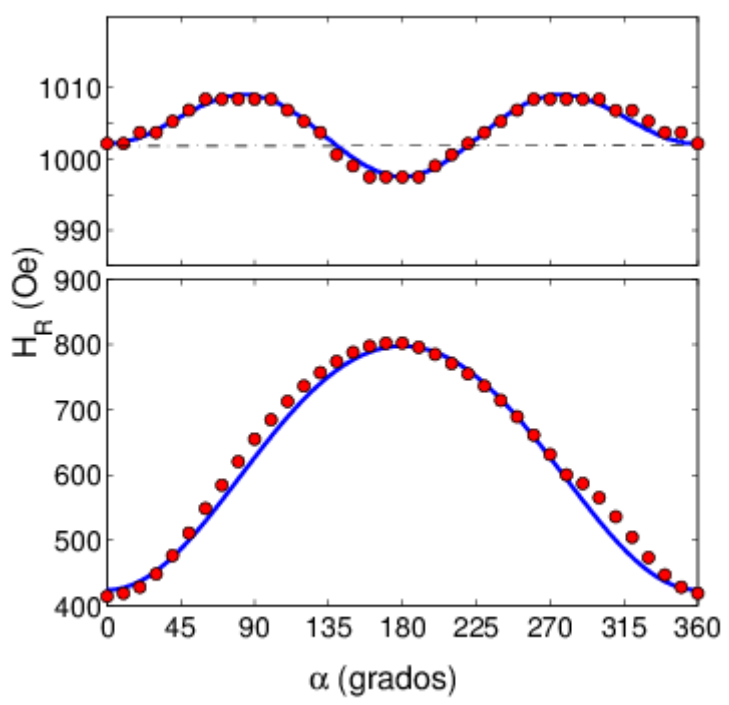

Figura 8: Dependencia angular $(\alpha)$ del campo de resonancia $H_{R}$ para el caso del acoplamiento antiferromagnético. Los puntos de color rojo son los datos experimentales tomados de la Ref. [17], la línea azul es el ajuste teórico para $J_{b l}+2 J_{b c}=-4.3$ $\mathrm{erg} / \mathrm{cm}^{2}$. 
Con toda esta información inicial, se resuelve numéricamente la Ec. (11) para cada valor del campo $H$, dentro de un rango específico y donde los ángulos de equilibrio son obtenidos al minimizar la función de densidad de energía, Ec. (7). Estos resultados se comparan a los datos experimentales mediante el cálculo del chi cuadrado $\chi^{2}$. Para ello se busca minimizar el $\chi^{2}$ haciendo variar los parámetros mencionados líneas arriba y utilizando el método numérico descrito en la Ref. [11]. Finalmente, la aplicación del modelo propuesto indica que para el espesor $t=32 \AA$ de la capa de Ru (capa espaciadora no-magnética), el acoplamiento es antiferromagnético, cuyo ajuste a los datos experimentales se muestra en la Fig. 8, Los parámetros óptimos que se obtuvieron al minimizar el $\chi^{2}$ se indican en la tabla 2

Allí se observa que los factores de acoplamiento bilineal y bicuadrático fueron los siguientes: $J_{b l}=-50.3 \times 10^{-3}$ $\mathrm{erg} / \mathrm{cm}^{2}$ y $J_{b c}=23.0 \times 10^{-3} \mathrm{erg} / \mathrm{cm}^{2}$. Cabe mencionar que la simulación se hizo con la simplificación de que $\phi_{1}=\alpha\left(\phi_{1}=\alpha+\pi\right)$ y $\phi_{2}=\alpha+\pi\left(\phi_{2}=\alpha\right)$ para la parte superior (inferior) de la fig. 8 Además, se considera el hecho de que $J_{b l}<0$ y $J_{b c}>0$ favorecen un alineamiento antiparalelo de $\boldsymbol{M}_{1}$ y $\boldsymbol{M}_{2}$, conforme al diagrama de fases elaborado en la Ref. [18].

\begin{tabular}{|c|c|c|c|c|c|c|}
\hline & \multicolumn{6}{|c|}{ Parámetros } \\
\hline Capa & $\begin{array}{c}t \\
(\stackrel{\circ}{A})\end{array}$ & $\begin{array}{c}H_{u} \\
(\mathrm{Oe})\end{array}$ & $\begin{array}{l}4 \pi M_{s} \\
(\mathrm{kOe})\end{array}$ & $\begin{array}{l}H_{K \text { eff }} \\
(\mathrm{kOe})\end{array}$ & $\begin{array}{c}J_{b l}+2 J_{b c} \\
10^{-3} \mathrm{erg} / \mathrm{cm}^{2}\end{array}$ & $\begin{array}{c}\gamma / 2 \pi \\
(\mathrm{GHz} / \mathrm{kOe})\end{array}$ \\
\hline $\mathrm{NiFe}$ & 50 & 4.4 & 10.0 & -10.0 & & $2.612(g=1.866)$ \\
\hline $\mathrm{Ru}$ & 32 & - & - & - & -4.3 & \\
\hline Co & 50 & 8.0 & 17.0 & -17.0 & & $2.640(g=1.886)$ \\
\hline IrMn & 150 & - & - & - & & \\
\hline
\end{tabular}

Tabla 2: Los parámetros mostrados en esta tabla han sido utilizados en el ajuste del sistema $\operatorname{IrMn}(150 \AA) / \mathrm{Co}(50 \AA) / \mathrm{Ru}(32 \AA) / \mathrm{NiFe}(50 \AA)$ el cual se muestra en la fig. 8 El campo de anisotropía de intercambio es $H_{E}=193$.

\section{Conclusiones}

En el presente trabajo se ha estudiado la resonancia ferromagnética de un sistema tipo válvula de espín y en particular se ha aplicado el modelo al sistema IrMn/Co/Ru/NiFe. Para ello se ha resuelto la ecuación de Landau-Lifhistz, la cual rige la dinámica de la magnetización alrededor de las posiciones de equilibrio de la magnetización. Luego se ha hecho el estudio de la relación de dispersión. La dependencia entre la frecuencia de resonancia y el campo aplicado está acorde con lo publicado en la literatura. El ajuste del modelo a los datos experimentales de campo resonante del sistema $\operatorname{IrMn} / \mathrm{Co} / \mathrm{Ru}(\mathrm{t}=32 \AA) / \mathrm{NiFe}$ muestra la existencia de un acoplamiento antiferromagné- tico con $J_{b l}+2 J_{b c}=-4.3 \mathrm{erg} / \mathrm{cm}^{2}$ y campo de anisotropía unidireccional de $H_{E}=193 \mathrm{Oe}$, con campos uniaxiales de $H_{u 1}=4.4 \mathrm{Oe} \mathrm{y} H_{u 2}=8 \mathrm{Oe}$, para la capa de NiFe y Co, respectivamente.

Esta metodología planteada aquí puede ser utilizada para el estudio de sistemas multicapas magnéticas del tipo presentadas en este trabajo.

\section{Agradecimientos}

Los autores agradecen al Dr. William Alayo del Centro Brasileiro de Pesquisas Físicas por habernos facilitado sus datos experimentales de resonancia ferromagnética.

\section{Referencias}

[1] B. Dieny, V. S. Speriosu, S. Metin, S. S. P. Parkin, B. A. Gurney, P. Baumgart y D. R. Wilhoit; J. Appl. Phys. 69, 4774 (1991).

[2] Ching Tsang, R. E. Fontana, Tsann Lin, D. E. Heim, Virgil S. Speriosu, B. A. Gurney y M. L. Williams; IEEE Trans. Mag. 30, 3801 (1994).

[3] D. Mauri, H. C. Siegmann, P. S. Bagus y E. Kay; J. Appl Phys. 62, 3047 (1987)
[4] J. Geshev, L. G. Pereira y F. E. Schmit, Phys. Rev. B 64, 184411 (2011)

[5] L. D. Landau y E. M. Lifshitz; To the theory of the dispersion of the ferromagnetic-body permeability, L. D. Landau Collected Papers, D. ter Haar, Editor, Pergamon, Oxford (1965)

[6] Michael Farle, Rep. Prog. Phys. 61, 755 (1998)

[7] Smit and H. G. Beljers, Philips Res. Rep. 10, 113 (1955) 
[8] S. M. Rezende, C. Chesman, M. A. Lucena, A. Azevedo, F. M. de Aguir y S. S. P. Parkin; J. Appl. Phys. 84, 958 (1998).

[9] A. Layadi, Phys. Rev. B 63, 174410 (2001).

[10] L. Rodríguez-Suárez, S. M. Rezende, and A. Azevedo, Phys. Rev. B 71, 224406 (2005).

[11] H. S. Tarazona, Estudio de los modos resonantes, Tesis de Licenciatura, UNMSM, Lima, Perú, (2014).

[12] Z. Zhang, L. Zhou y P. E. Wigen, Phys. Rev. B 50, 9 (1994).

[13] A. Layadi, Phys. Rev. B 72, 024444 (2005)
[14] J. Lindner y K. Baberschke, J. Phys. Condens. Matter 15, S465 (2003).

[15] Klaus Baberschke, Handbook of Magnetism and Advanced Magnetic Materials Vol. 3, 1627, H. KronmüIler and S. S. Parkin, Eds, John Wiley \& Sons, Ltd., New York (2007)

[16] L. Rodríguez-Suárez, S. M. Rezende y A. Azevedo, Phys. Rev. B 71, 224406 (2005).

[17] W. Alayo, M. A. Souza, F. Pelegrini y E. BaggioSaitovitch, J. Appl. Phys. 109, 83917 (2011).

[18] J. C. Slonczewski, J. Magn. Magn. Mater. 150, 13 (1995) 